\title{
Cortical Surface Registration Using Texture Mapped Point Clouds and Mutual Information
}

\author{
Tuhin K. Sinha, David M. Cash, Robert J. Weil, \\ Robert L. Galloway, and Michael I. Miga \\ Vanderbilt University, Nashville TN 37235, USA \\ \{tk.sinha, dave.cash, michael.i.miga\}@vanderbilt.edu \\ robert.j.weil@mcmail. vanderbilt.edu \\ http://bmlweb. vuse.vanderbilt.edu
}

\begin{abstract}
An inter-modality registration algorithm that uses textured point clouds and mutual information is presented within the context of a new physical-space to image-space registration technique for imageguided neurosurgery. The approach uses a laser range scanner that acquires textured geometric data of the brain surface intraoperatively and registers the data to grayscale encoded surfaces of the brain extracted from gadolinium enhanced MR tomograms. Intra-modality as well as inter-modality registration simulations are presented to evaluate the new framework. The results demonstrate alignment accuracies on the order of the resolution of the scanned surfaces (i.e. submillimetric). In addition, data are presented from laser scanning a brain's surface during surgery. The results reported support this approach as a new means for registration and tracking of the brain surface during surgery.
\end{abstract}

\section{Introduction}

Understanding the geometric characteristics and the impact of intraoperative surgical events upon the cortical brain surface has important implications in the development of image-guided surgery (IGS) systems. In recent studies 1, the need for brain shift compensation strategies to prevent compromising IGS navigation has become an important area of research [2. When using a computational approach to correct for brain shift [3, capturing the geometric and visual changes of the brain surface due to deformation may be a valuable source of intra-operative data. To achieve this end, a laser range scanning system capable of capturing textured surfaces with sub-millimetric accuracy will be used. Using features from the cortical surface to register does have precedent. Nakajima et al. demonstrated an average of $2.3 \pm 1.3 \mathrm{~mm}$ fiducial registration error (FRE) using cortical vessels for registration [4]. More recently, Nimsky et al. reported a deformable surface approach to quantify surface shifts using a variation on the iterative closest point (ICP) algorithm [1]. Also, some preliminary work utilizing a scanning based system for cortical surface registration has been reported but a systematic evaluation has not been performed to date [5]. The novelty of the approach reported here is that both vessel information and three-dimensional

T. Dohi and R. Kikinis (Eds.): MICCAI 2002, LNCS 2489, pp. 533-540 2002.

(C) Springer-Verlag Berlin Heidelberg 2002 
topography will be used as the basis of alignment. Furthermore, the scanner provides a highly accurate method for tracking the brain surface that can be used in the model-updating framework.

As an initial step, an implementation has been developed using an iterative closest point (ICP) [6] framework with mutual information (MI) [7]. Although ICP and MI have been used extensively [ 8] [9], previously published registration frameworks do not entirely apply to the unique data provided by the scanner or this particular registration approach. The data acquired by the scanner provides a one-to-one correspondence between contour point and image intensity. However, intensity correspondence between a three-dimensional MR surface and an intraoperatively acquired laser-scanned cortical surface is somewhat more elusive. The most similar work relating to this registration framework is that by Johnson and Kang [10] in which these investigators used an objective function for registration based on a combined Euclidean distance and color difference metric. Used primarily in a landscape alignment application, this technique would not be amenable to the alignment process here, since the intensity distribution between scanner and MR image data is fundamentally very different. To our knowledge, no registration algorithm has been developed that will register textured three-dimensional surfaces from two different imaging modalities within the context of cortical surface registration.

\section{Methods}

In the realization of this approach, a laser range scanning system (RealScan 3D, 3D Digital Corporation, Danbury, CT) capable of capturing three-dimensional textured surfaces to sub-millimeter accuracy has been utilized (see Figure 1). The scanner is lightweight, compact and has a standard tripod mount. The scanning field consists of 500 horizontal by 494 vertical points per scan and is accomplished in approximately 5 seconds. Extensive calibration and characterization has been performed by Cash et al. and has demonstrated the fidelity at which surface data can be acquired [11]. Additionally, the device is approved for use in neurosurgery by the Vanderbilt University Medical Center Institutional Review Board.

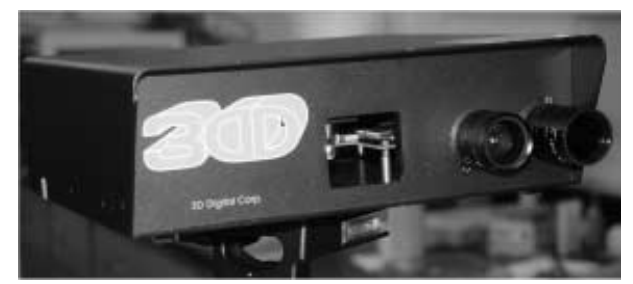

Fig. 1. Laser scanner used to acquire textured point clouds.

The registration framework involves two primary steps in its execution. The first step involves acquisition and preparation of the registration surfaces. With 
respect to laser scanned surfaces, the scanner is currently placed approximately 1-2 feet from the surface of interest (achieved either by passive arm or monopod for intraoperative use). The horizontal range of the scanner is established and a vertical laser stripe passes over the surface in approximately 5 seconds. The data acquired consists of a three-dimensional point cloud with each Cartesian coordinate color-encoded via texture mapping into a digital image that is acquired just after scanning. The texture-space to scanner-space registration is calibrated by the manufacturer. The MR-generated point cloud is prepared by segmenting the brain volume, followed by ray-casting to find surface points, and averaging subsequent voxels to generate gray-scale values for each surface point (Analyze AVW - Biomedical Imaging Resource).

The final step in our approach is to perform surface registration using a twostage process. An iterative closest point (ICP) algorithm is performed initially to align the point clouds of interest (i.e. laser-scanned surface and/or MR surface). The second stage is a constrained intensity-based registration. The constraint requires the alignment transformation to only operate in spherical coordinates with known radius $R$; the radius is provided by sphere-fitting the target surface [12. By enforcing this restriction on the transformation, the degrees of geometric freedom are reduced from six to three, i.e. elevation $\phi$, azimuthal $\theta$, and roll $\psi$. For the method of intensity-based registration, a maximization of normalized mutual information (NMI) [13] approach is conducted using Powell's optimization algorithm 14. Referred to as Surface MI in this work, the method aligns textured surfaces only and does not use volumetric image data. The results presented here do not reflect true cross-modality registration (i.e. scanner to MR).

\section{$3 \quad$ Registration Experiments}

To evaluate robustness and accuracy of Surface MI, an initial series of experiments was conducted using a spherical phantom with a heterogenous intensity pattern on the surface. The range scanned surface acquired for registration experiments occupied a solid angle of $\Omega=1.2 \pi$ steradian 11 and contained 67257 points (see Figure 2). A known transformation was then applied to the target surface to generate the floating surface. The 1 imits for elevation, azimuthal and roll angle perturbations were $\pm 13, \pm 13$, and \pm 25 degrees, respectively (the radius of the spherical phantom was approximately $110 \mathrm{~mm}$ ). The floating and target surfaces are then re-registered using Surface MI. Five hundred randomly distributed combinations of $\phi, \theta$, and $\psi$ were tested for registration accuracy.

The second series of experiments employed the point clouds generated from surface projections of the MR volume. The target surface that was generated using a clipping plane had a solid angle of approximately $\Omega=.38533 \pi$ steradians and contained 48429 points (see Figure 3). Similar to the spherical phantom experiments, perturbations in $\phi, \theta$, and $\psi$ were applied to the MR surface over 500 trials. The range for the parameters $\phi, \theta$, and $\psi$ were the same as those for $\mathrm{t}$ he previous experiment with similar radius $(R=105 \mathrm{~mm})$.

\footnotetext{
${ }^{1}$ The solid angle of a unit sphere $\Omega=4 \pi$ steradians.
} 

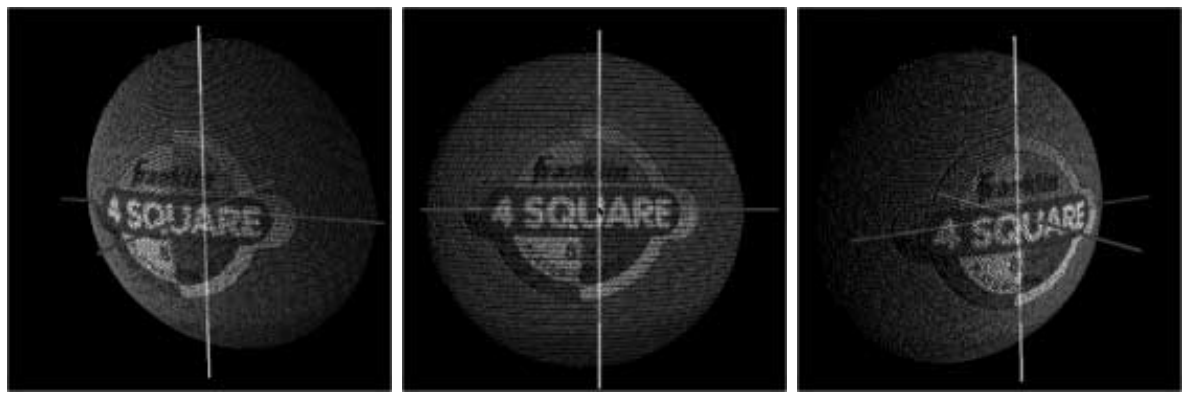

Fig. 2. Sample textured point cloud generated using a laser range scanner.
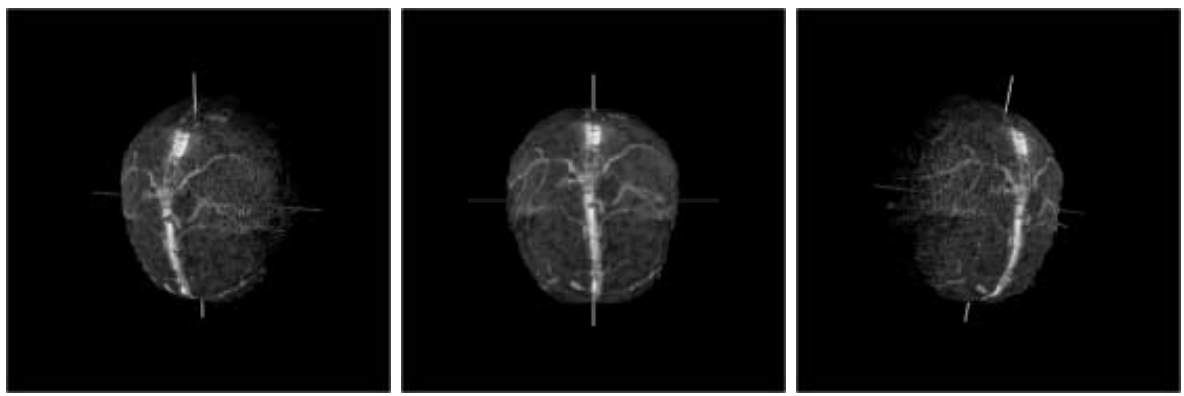

Fig. 3. Sample textured point cloud generated using surface projection on a gadolinium enhanced MR volume.

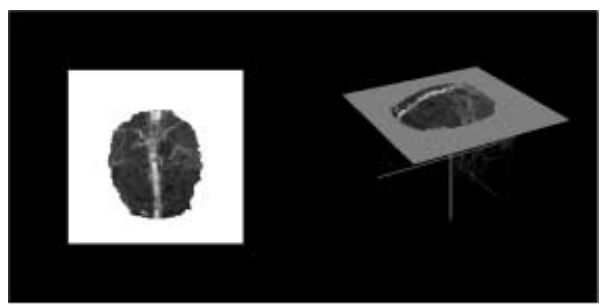

Fig. 4. Use of a clipping plane to select a region of interest in the surface projection.

The last series of experiments evaluated the efficacy of the developed algorithm in registering surfaces across modalities. Inter-modality surfaces were simulated by inverting the texture of the point cloud. Five hundred trials registering a texture-inverted region of interest (ROI) to the original MR brain surface were performed with initial misregistrations comparable to the spherical phantom experiments. The ROIs were generated by varying the normal of the clipping plane used to create the target sur face between $\pm 0.1 \mathrm{~cm}$ in the sagittal and coronal axis while holding the axial value at $1 \mathrm{~cm}$ (see Figure 4 ). To create the misregistration between the float and target surface, each surface was re-centered about it's geometric centroid. 


\section{Registration Results and Discussion}

Since the same scan was used for both target and floating surfaces in the registrations process, the one-to-one correspondence in points was known. This allowed calculation of the mean target registration error (TRE) between point clouds as well as the global maximum for NMI. Sample registration results are presented for each experiment series (i.e. spherical phantom, intra-modality MR, simulated inter-modality MR) in Figure 5. In addition, a distribution of TREs for each series of experiments can be seen in Figure 6 .

Registration results from the 500 trials using the spherical phantom yielded a mean TRE of $11.38 \pm 28.75 \mathrm{~mm}$ (min. $=0.04$, $\max .=127.61 \mathrm{~mm}$ ). Although this result is less than remarkable, it should be noted that $70 \%$ of the trials achieved a mean TRE of $0.20 \pm 0.05 \mathrm{~mm}$ (min. $=0.04, \max .=0.31 \mathrm{~mm}$ ). Furthermore, the misalignment range during surgery is expected to be \pm 5 degrees within each angular coordinate. Within this range, the registration process achieved a $100 \%$ success rate (i.e. NMI optimization reached it's global maximum).

With respect to the intra-modality MR experiments, all 500 trials resulted in an ideal value of NMI. The mean TRE for the 500 trials was $0.14 \pm 0.04 \mathrm{~mm}$ $(\min .=0.04, \max .=0.27 \mathrm{~mm})$. The increased success rate of this series of experiments as compared to the previous trials is likely due to the differences in the geometric structure of the intensity information. Most of the intensity information of the spherical phantom is contained in the central region of the surface. In some cases, when the initial mis-registration of the spherical phantom caused sufficient non-overlap of the central area, the algorithm did not register the surfaces correctly. For the brain, the intensity pattern of the vessel structure occupies most of the surface. Thus, even though the brain's surface occupies a smaller solid angle than that of the ball, the distribution of the intensity pattern allows the alignment of more severely misregistered surfaces.

The last series of experiments simulating inter-modality registration generated a mean TRE of $3.38 \pm 7.18 \mathrm{~mm}$ (min. $=0.07, \max .=53.75 \mathrm{~mm}$ ). Similar to the spherical phantom, $67 \%$ of these trials produced a mean TRE of $0.37 \pm 0.19 \mathrm{~mm}$ $(\min .=0.07, \max .=1.00 \mathrm{~mm})$. Analysis of the failed trials indicated that the spherical constraint prevented accurate registration. In general, the algorithm failed to register surfaces clipped from or containing the periphery of the surface projection, which contained a much higher surface curvature as compared to the target surface. This discrepancy in surface curvatures between target and floating surfaces caused the sub-optimal registrations. In general, the occurrence of curvature discrepancies intra-operatively will be limited since vessel landmarks will be used to provide an initial alignment for the Surface MI.

\section{Conclusions and Future Work}

The results of this paper show that the ICP and MI framework is a useful tool for cortical surface registration. Results of both intra- and inter-modality surface registration show sub-millimetric accuracies using a phantom. This paper outlines preliminary steps taken with the laser range scanner and the Surface 


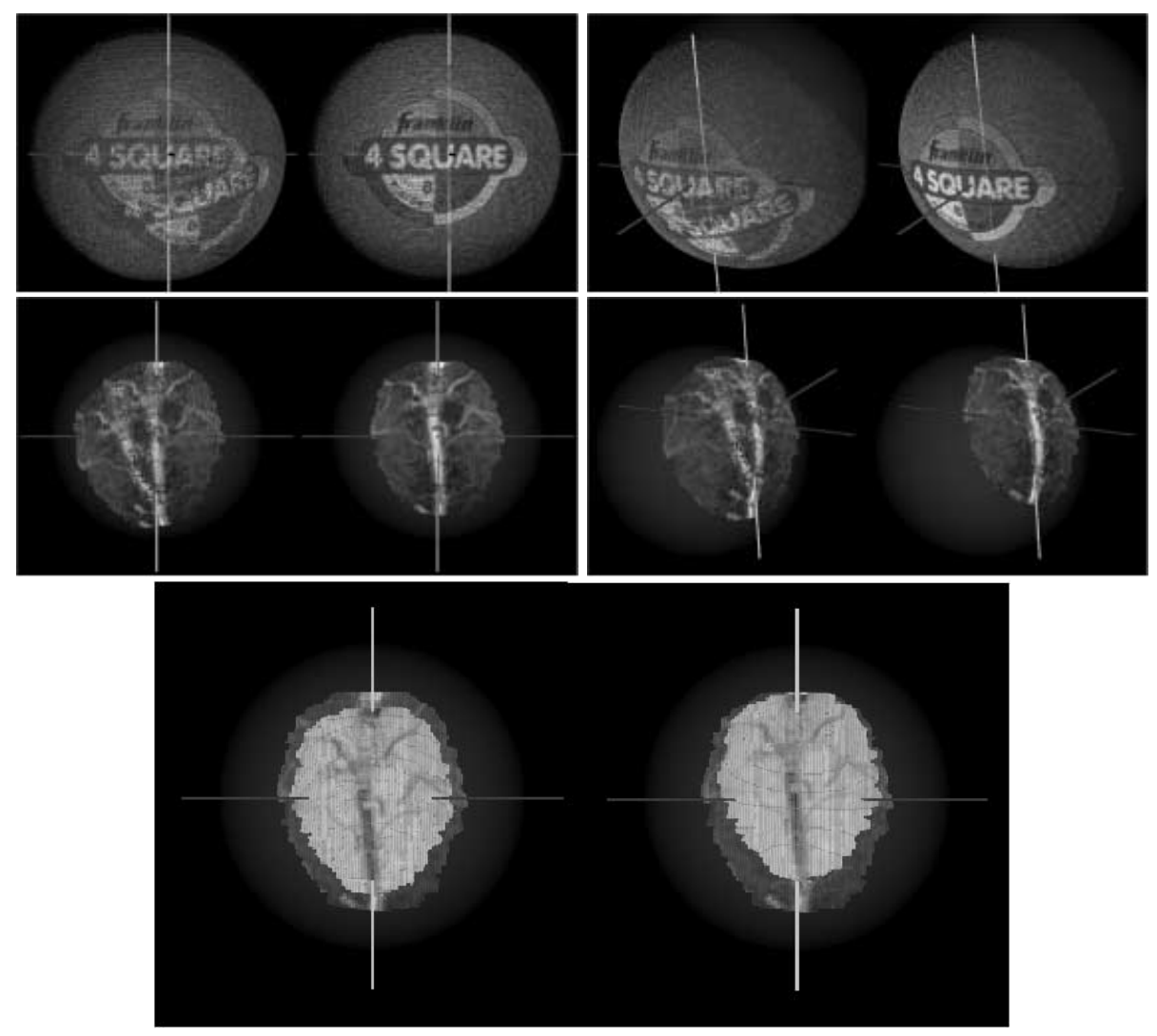

Fig. 5. Sample registration results. Top row, from left to right: on-axis view of misregistered and registered surfaces of the spherical phantom, off-axis view of misregistered and registered surfaces. Middle row: sample results of the intra-modality registration, presented similar to the top row. Bottom row from left to right: misregistered and registered surfaces from simulated inter-modality experiments.

MI algorithm. In vivo analysis of the registration results is currently in progress. Figure 7 shows intra-operative data of the cortical surface acquired by the laser range scanner. More quantitative studies of the laser range scanner and registration algorithm are also planned using an optical tracking system. Algorithmically, the ability to track and register cortical deformations is also being studied.

\section{Acknowledgements}

The authors acknowledge Dr. Hill for his correspondence on MI. VTK (Kitware Inc.) and Analyze AVW (Mayo Clinic) provided software. A grateful acknowledgement to the VUMC Neurosurgical staff. This project is supported in part by the Vanderbilt University Discovery Grant Program. 


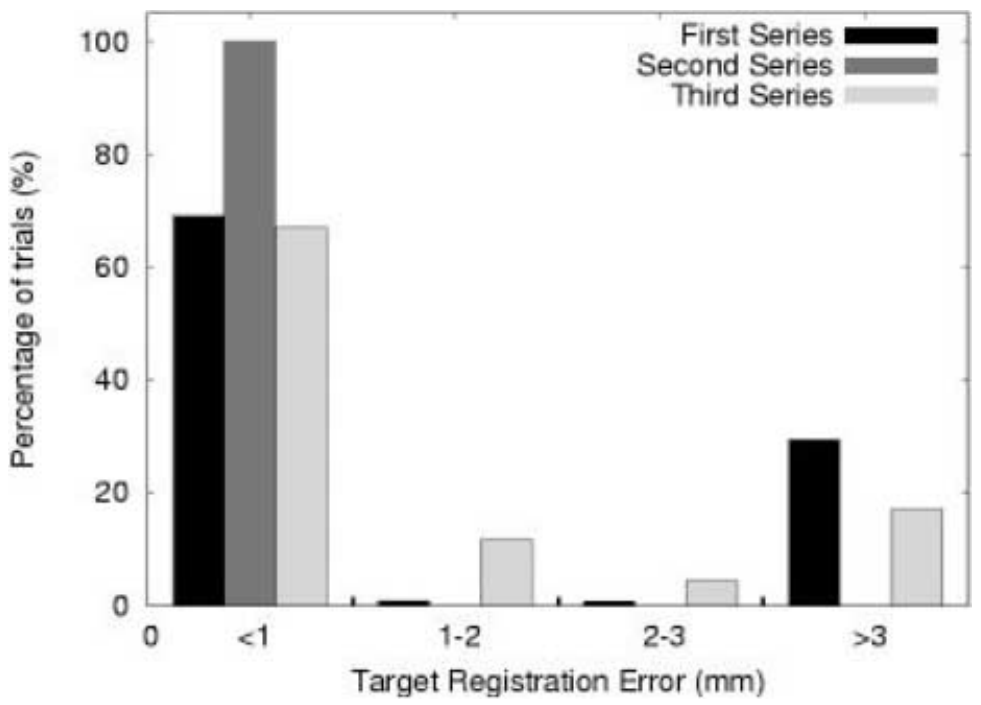

Fig. 6. Distribution of Target Registration Error (TRE) for each series of experiments.

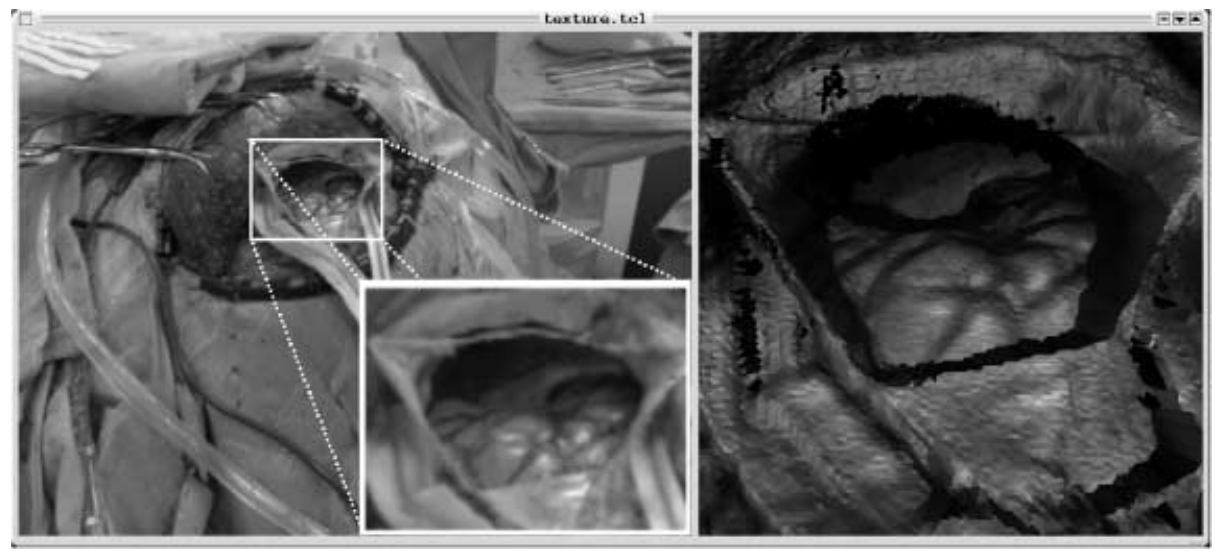

Fig. 7. Example dataset taken with the laser range scanner in the operating room. Left, a CCD image of the surgical area. Right, a tessellated point cloud with texture mapped points on the right.

\section{References}

1. Nimsky, C., Ganslandt, O., Cerny, S., Hastreiter, P., Greiner, G., Fahlbusch, R.: Quantification of, visualization of, and compensation for brain shift using intraoperative magnetic resonance imaging. Neurosurgery 47 (2000)

2. Roberts, D., Miga, M., Hartov, A., Eisner, S., Lemery, J., Kennedy, F., Paulsen, K.: Intraoperatively updated neuroimaging using brain modeling and sparse data. Neurosurgery 45 (1999) 
3. Miga, M., Paulsen, K., Lemery, J., Eisner, S., Hartov, A., Kennedy, F., Roberts, D.: Model-updated image guidance: Initial clinical experiences with gravity-induced brain deformation. IEEE: Trans. on Med. Img. 18 (1999)

4. Nakajima, S., H, H.A., Kikinis, R., Moriarty, T.M., Metcalf, D.C., Jolesz, F.A., Black, P.M.: Use of cortical surface vessel registration for image-guided neurosurgery. Neurosurgery 40 (1997)

5. Audette, M.A., Siddiqi, K., Peters, T.M.: Level-set surface segmentation and fast cortical range image tracking for computing intrasurgical deformations. LNCS: Med. Image Computing and Computer-Assisted Intervention 1679 (1999)

6. Besl, P.J., McKay, N.D.: A method for registration of 3-d shapes. IEEE Trans. on Pattern Analysis and Machine Intelligence 14 (1992)

7. Wells, W.M., Viola, P., Atsumi, H., Nakajima, S., Kikinis, R.: Multi-modal volume registration by maximization of mutual information. Med. Image Analysis 1 (1996) $35-51$

8. Maes, F., Collignon, A., Vandermeulen, D., Marchal, G., Suetens, P.: Multimodality image registration by maximization of mutual information. IEEE Trans. on Med. Imag. 16 (1997) 187-198

9. Audette, M.A., Ferrie, F.P., Peters, T.M.: An algorithmic overview of surface registration techniques for med. imag.. Med. Image Analysis 4 (2000) 201-217

10. Johnson, A.E., Kang, S.B.: Registration and integration of textured 3d data. Image and Vision Computing 17 (1999) 135-147

11. Cash, D.M., Sinha, T.K., Chapman, W.C., Galloway, R.L., Miga, M.I.: Fast accurate surface acquisition using a laser scanner for image-guided surgery, SPIE: Med. Imag. 2002 (2002)

12. Ahn, S.J., Rauh, W., Warnecke, H.J.: Least-squares orthogonal distances fitting of circle, sphere, ellipse, hyperbola, and parabola. Pattern Recognition 34 (2001)

13. Studholme, C., Hill, D.L.G., Hawkes, D.J.: An overlap invariant entropy measure of 3d medical image alignment. Pattern Recognition 32 (1999) 71-86

14. Press, W.H., Teukolsky, S.A., Vetterling, W.T., Flannery, B.P.: Num. Rec. in C : The Art of Scientific Computing. Second edn. Cambridge University Press (1993) 